\title{
Le flux numérique en implantologie
}

\section{Daas (Paris), K. Dada (Paris)}

L'optimisation du résultat esthétique et fonctionnel des restaurations implantaires, la simplification des procédures cliniques et de laboratoire et l'amélioration de la prévisibilité des traitements sont les principaux objectifs de l'implantologie moderne.

Cette prévisibilité est due en grande partie à l'intégration du flux numérique dans les différents étapes du traitement: analyse esthétique virtuel « Digital Smile Design », meilleure communication avec les patients, prise en compte des paramètres biologiques, esthétiques et fonctionnels, prise d'empreinte optique, numérisation des modèles, planification implantaire 3D, chirurgie guidée, optimisation du profil d'émergence avec les piliers anatomiques personnalisés, mise en place d'une restauration implanto-portée se rapprochant le plus possible des dents naturelles absentes, en se fondant dans son environnement tout en respectant la notion de «Biomimétique». Cette démarche permet donc aujourd'hui d'anticiper le résultat de nos traitements, devenus de plus en plus complexes, et d'assurer la pérennité de nos restaurations implantaires à long terme.

Le but de notre intervention est de présenter l'intégration de ces nouvelles technologies dans notre pratique quotidienne, du cas simple au plus complexe.

Marwan DAAS - 62 BD de la Tour Maubourg - 75007 PARIS - 0610284494 cabinet.marwann@wanadoo.fr 